\title{
Home, Clinic, and Ambulatory Blood Pressure Monitoring in Children with Chronic Renal Failure
}

\author{
ELKE WÜHL, CHARLOTTE HADTSTEIN, OTTO MEHLS, FRANZ SCHAEFER, \\ AND THE ESCAPE TRIAL GROUP* \\ Division of Pediatric Nephrology, University Children's Hospital, University of Heidelberg, Im \\ Neuenheimer Feld 151, 69120 Heidelberg, Germany
}

\begin{abstract}
Ambulatory blood pressure monitoring (ABPM) provides superior information for diagnosis and treatment of pediatric hypertension, but for reasons of practicality, clinic blood pressure measurements (CBP) are still the primary diagnostic tool. Regular home blood pressure measurements (HBP) may be an alternative to $A B P M$, but this technique awaits validation in practice. We analyzed the concordance of ABPM, CBP and HBP in 118 pediatric patients (3-19 y) with chronic renal failure. HBP readings (10.5 \pm 5.4 per patient) were averaged for one week around the day of ABPM and CBP. Mean arterial pressure (MAP) measured by HBP $(84.0 \pm 10 \mathrm{~mm} \mathrm{Hg})$ was significantly lower than both CBP $(86.1 \pm 14.1 \mathrm{~mm} \mathrm{Hg}, P<0.05)$ and daytime ABPM $(90.3 \pm 10.4 \mathrm{~mm} \mathrm{Hg}, P<0.05)$. HBP detected hypertensive patients with greater specificity (82 versus $70 \%$ ), but lower sensitivity (52 versus $70 \%$ ) than CBP. The fraction of patients rated erroneously hypertensive was $23 \%$ with CBP, but only $14 \%$ with HBP. The $95 \%$ limits of agreement with ABPM
\end{abstract}

\section{ABSTRACT}

were narrower for HBP (-23 to $10 \mathrm{~mm} \mathrm{Hg}$ ) than for CBP (-30 to $21 \mathrm{~mm} \mathrm{Hg}$ ). CBP, but not HBP measurements, were less precise in the upper BP range. The accuracy of HBP measurements did not change with use over a six months time period. In conclusion, HBP was superior to CBP in predicting ABPM, but neither CBP nor HBP detected hypertension with enough sensitivity or specificity to replace ABPM. The greater specificity of HBP compared with CBP makes it a more suitable tool for diagnosis, rather than screening, of hypertension in children. (Pediatr Res 55: 492-497, 2004)

\section{Abbreviations}

ABPM, ambulatory blood pressure monitoring BP, blood pressure

CBP, clinic (also casual or office) blood pressure

HBP, home blood pressure

MAP, mean arterial pressure
In patients with chronic renal failure (CRF) hypertension is not only a general cardiovascular risk factor, but is also increasingly recognized as a crucial determinant of the rate of progression of renal disease to end-stage renal failure $(1,2)$. Therefore, close monitoring of blood pressure (BP) is an important part of the care of patients with CRF. Twenty-four hour ambulatory blood pressure monitoring (ABPM) has been found to be superior to clinic BP measurements (CBP) in predicting end organ damage (3) and useful for the detection of nocturnal BP changes, which are predictive of cardiovascular mortality in CRF (4). In adults ABPM has become generally accepted as the gold standard for the diagnosis and therapeutic

Received July 10, 2003; accepted September 9, 2003.

Correspondence: Franz Schaefer, M.D., University Children's Hospital, Im Neuenheimer Feld 151, 69120 Heidelberg, Germany; e-mail: franz_schaefer@med.uni-heidelberg.de.

Support for this study was obtained from the European Union (5th Framework Programme, QLG1-CT-2002-00908), the Boehringer Ingelheim Foundation, the Baxter Extramural Grant Program, and Aventis Pharma.

*See Appendix for list of members.

DOI: 10.1203/01.PDR.0000106863.90996.76 monitoring of renal hypertension. However, the considerable costs and organizational effort involved with this method limit the routine use of ABPM. Self-measurements of BP at home (HBP) have been advocated in place of ABPM in adults and small cross-sectional studies have suggested that the correlation of HBP to end organ damage may be as good as that of ABPM (5). However, the validity of HBP relative to ABPM and routine CBP has not been assessed systematically in patients with renal hypertension, and is entirely unknown for the pediatric setting, where HBP measurements are usually performed by the parents.

To investigate whether HBP measurements provide a feasible and reliable alternative to ABPM in differentiating true from white-coat hypertension and in monitoring antihypertensive therapy in children, we analyzed the concordance of concomitant clinic, HBP and ABPM readings in a large pediatric CRF patient cohort by both cross-sectional and longitudinal assessment.

\section{METHODS}

Patients. Concomitant ABPM, CBP and HBP measurements were obtained in children treated in 33 pediatric nephrology 
units in 13 European countries. The children were monitored as part of an ongoing prospective multicenter trial (ESCAPE (Effect of Strict Blood Pressure Control and ACE Inhibition on the Progression of CRF in PEdiatric Patients) Study). All children suffered from mild to moderate CRF. Mean 24-h systolic blood pressure was above the 50th percentile for height and/or controlled by antihypertensive medication. Further patient characteristics are given in Table 1.

In the ESCAPE trial, all patients receive a fixed dose of the ACE inhibitor ramipril $\left(6 \mathrm{mg} / \mathrm{m}^{2}\right.$ daily) and are additionally randomized to either intensified BP control aiming at a 24-h mean arterial BP below the 50th percentile or conventional antihypertensive treatment accepting BP in the high normal range (i.e. 50th to 95 th percentile). ABPM is performed before and after a six-month run-in period, and every six months after start of ramipril and randomization to intensified or conventional BP control. In addition, CBP is measured at two monthly intervals, and daily HBP measurements are optionally performed. The ESCAPE study is an investigator initiated trial; the study design and the collection, analysis and interpretation of the data are carried out independently of the financial supporters. The study has been approved by the ethical committees of all participating centers and informed consent was obtained from the parents of each of the subjects.

In 118 patients sufficient HBP data, defined by at least three home measurements during two to 7 days around an ABPM profile and concomitant clinic visit, were available on one or several occasions. The first triplet of ABPM, HBP and CBP measurements was usually obtained at the screening or randomization visit. In 70 patients, one additional combined reading was obtained at least six months later (mean $7.7 \mathrm{mo}$ ) and used for longitudinal analysis of the accuracy and precision of HBP. In a further analysis CBP was also averaged with two additional clinic readings taken two months before and after

Table 1. Patient characteristics

\begin{tabular}{lcc}
\hline & $\mathrm{N}$ & $\%$ \\
\hline Total & 118 & \\
Sex (male) & 68 & 58 \\
Cause of renal failure & & \\
$\quad$ Renal hypo/dysplasia & 86 & $74 \%$ \\
$\quad$ Hereditary and congenital nephropathies & 15 & $13 \%$ \\
$\quad$ Acquired glomerulopathies & 16 & $14 \%$ \\
$\quad$ Number of antihypertensive drugs & & \\
$\quad$ None & 26 & $22 \%$ \\
$\quad$ One & 57 & $48 \%$ \\
$\quad$ Two & 24 & $20 \%$ \\
$\quad$ More than three & 11 & $10 \%$ \\
\hline & Mean $\pm \mathrm{SD}$ & $\mathrm{Range}$ \\
\hline Age (years) & $11.2 \pm 4$ & $3-19$ \\
Height (cm) & $141 \pm 22$ & $98-187$ \\
Height SDS & $-0.7 \pm 1.4$ & $-5-3$ \\
BMI (kg/m ${ }^{2}$ ) & $18 \pm 3$ & $13-27$ \\
BMI SDS & $-0.02 \pm 1.3$ & $-3-3$ \\
24 hour MAP (mmHg) & $86 \pm 10$ & $67-115$ \\
24 hour MAP SDS & $0.5 \pm 1.6$ & $-2.3-7$ \\
daytime MAP (mmHg) & $90 \pm 10$ & $67-127$ \\
daytime MAP SDS & $0.7 \pm 1.6$ & $-2.3-7$ \\
Glomerular filtration rate $\left(\mathrm{ml} / \mathrm{min} / 1.73 \mathrm{~m}^{2}\right.$ ) & $53 \pm 23$ & $10-118$ \\
\hline
\end{tabular}

the original examination, in agreement with current recommendations for the diagnosis of hypertension (6). This analysis was performed in 63 patients whose antihypertensive treatment had remained unchanged for three subsequent CBP readings.

Blood pressure measurements. For the present study HBP measurements taken in the same week as a clinic visit were used. Between 3 and 21 measurements (mean $10.5 \pm 5.4$ per patient) were available for a time period of two to $7 \mathrm{~d}$ (mean 5 \pm 1.8 d). Morning HBP were available in $89 \%$, evening readings in $83 \%$ and midday readings in $61 \%$. Semi-automatic OMRON MX 1 BP devices, which have been validated for use in children (7), were provided to all families with the more appropriate of two available cuff sizes. At the outpatient visit, CBP was taken by a health care professional and the 24-hour ABP measurement was started. Of the clinic readings, 80 $(68 \%)$ were taken by auscultation with a standard sphygmomanometer and $38(32 \%)$ with an automatic oscillometric device (Dinamap) (7). ABPM was performed with a Spacelabs 90207 automatic cuff-oscillometric device. Measurements were obtained every $15 \mathrm{~min}$ during the day and every $30 \mathrm{~min}$ at night. For further analysis ABPM profiles were divided into daytime (08:00 to $20: 00 \mathrm{~h})$ and nighttime periods (24:00 to 06:00 h).

Unless otherwise stated all blood pressure measurements are given as mean arterial pressure (MAP), as this is measured directly by the ABPM device, while systolic and diastolic values are calculated via mathematical algorithms. For CBP and HBP MAP was calculated according to the following formula: MAP= diastolic BP $+[($ systolic $\mathrm{BP}-$ diastolic BP $) / 3]$.

Hypertension was defined for each method as either systolic or diastolic BP above the 95th percentile in relation to the child's gender and height, using the European reference values of Wühl et al. (8) for ABPM and those of de Man et al. (9) for CBP and HBP.

White coat effect (WCE) was defined as the absolute difference between daytime ABPM and CBP, while white coat hypertension (WCH) was defined as a CBP over the 95th percentile in the presence of daytime ABPM below the 95th percentile. For all calculations of sensitivity and specificity ABPM was used as the reference method.

Statistical analysis. ABPM data were analyzed with the Spacelabs ABPM Report Manager System and descriptive statistics were computed with the ABPM-fit and CV-sort software (10). Data were stored and processed using the SAS software (SAS Institute, Cary, NC, U.S.A.). Values are given as means $\pm \mathrm{SD}$. For continuous variables, correlations are expressed as Spearman's correlation coefficients and group comparisons were performed using Student's $t$ tests. For comparisons of categorical variables the $\chi^{2}$ analysis was used. Bland Altman plots (11) were used to illustrate the variation between HBP or CBP and ABPM measurements.

\section{RESULTS}

\section{Accuracy and precision of ABPM prediction by CBP and HBP}

The accuracy and precision of the prediction of the ABPM mean arterial pressure (MAP) by CBP and HBP is given in 
Table 2. All CBP and HBP measurements were significantly lower than daytime ABPM and higher than nighttime ABPM $(p<0.01)$, but only HBP varied significantly from 24 hour ABPM. Morning, midday and evening HBP did not differ significantly between each other, but were all lower than CBP $(p<0.05)$.

Twenty-four-hour and daytime ABPM MAP were predicted with similar precision by the mean of all available HBP measurements, the mean morning HBP and a single CBP reading ( $r=0.54$ to 0.71 , see Table 2 ). Midday and evening HBP measurements alone had a weaker relationship to ABPM than morning HBP or CBP. As expected from methodological differences, evening HBP was higher than nocturnal BP assessed by ABPM (by $6.7 \pm 11 \mathrm{~mm} \mathrm{Hg}$ ), and there was only a weak association of the two $(r=0.48)$, indicating that evening HBP cannot be used as a surrogate for the circadian BP regulation at night.

The accuracy and precision of $\mathrm{HBP}$ and $\mathrm{CBP}$ in predicting ABPM is further illustrated by Bland Altman plots (Fig. 1). The $95 \%$ confidence limits (i.e. $\pm 2 \mathrm{SD}$ ) encompass a range of $33 \mathrm{~mm} \mathrm{Hg}$ for HBP compared with $51 \mathrm{~mm} \mathrm{Hg}$ for CBP. Daytime ABPM tended to be systematically underpredicted by both HBP (mean $-6 \mathrm{~mm} \mathrm{Hg}$ ) and CBP (mean $-4 \mathrm{~mm} \mathrm{Hg}$ ). The mean between CBP and daytime ABPM was not significantly different for the two different methods $(-3.7 \pm 12 \mathrm{~mm}$ $\mathrm{Hg}$ for auscultatory and $-4.4 \pm 14 \mathrm{~mm} \mathrm{Hg}$ for oscillometric measurements).

Interestingly, even though CBP was lower than ABPM when all measurements were taken into account, it tended to overestimate ABPM in the upper BP range. This was confirmed by a significant linear regression fitting the CBP-ABPM Bland Altman plot $(r=0.35, p<0.0001)$ but not the plot comparing $\mathrm{HBP}$ and $\mathrm{ABPM}$.

Figure $2 A$ shows that the residual difference between daytime ABPM and CBP, which approximates the white coat effect (WCE) unadjusted for age and height, was well correlated with the prevailing CBP $(r=0.67, p<0.0001)$. In contrast, the WCE was almost independent of daytime ABPM $(r=-0.18, p=0.06)$ and 24-hour ABPM readings $(r=$ $-0.12, p=0.2)$. This phenomenon was not dependent on the method used to measure CBP, as the regression between the WCE and CBP did not differ significantly for the auscultatory and oscillometric readings (regression lines shown in Fig. 2A). The WCE was also correlated weakly but significantly with age
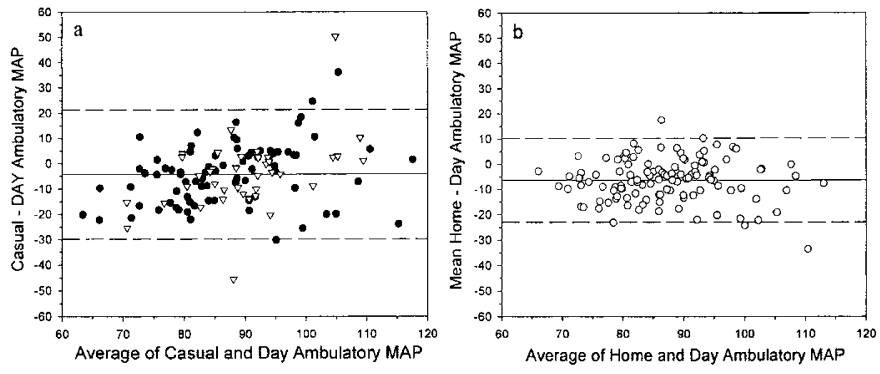

Figure 1. $(A$ and $B)$ Bland Altman plots showing the variance between $\mathrm{ABPM}$ and $\mathrm{CBP}$ (left panel) or HBP (right panel). All values as mean arterial pressure in $\mathrm{mm} \mathrm{Hg.} \mathrm{-} \mathrm{-} \mathrm{mean}( \pm 2 \mathrm{SD})$; ----, limits of confidence; $\bullet$, auscultatory measurements; $\nabla$, oscillometric measurements (Dinamap); $\circ$, oscillometric measurements (Omron).
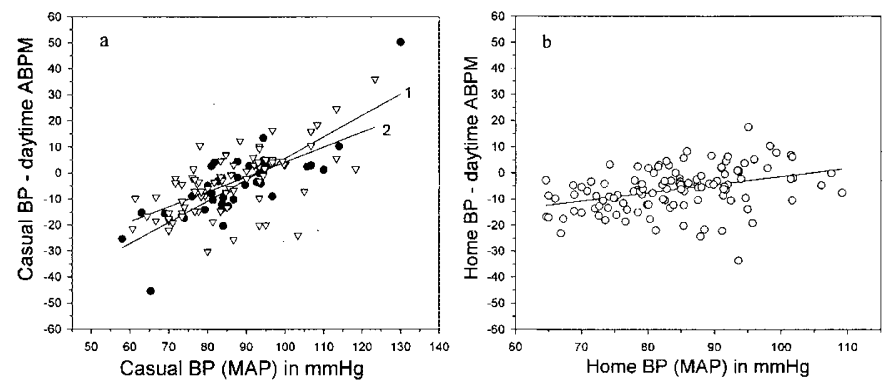

Figure 2. ( $A$ and $B$ ) Correlation of CBP (left panel) or HBP (right panel) to their difference from ABPM., CBP measurements (Auscultatory); $\nabla, \mathrm{CBP}$ measurements (Dinamap); $\odot$, HBP measurements (Omron); 1, regression line (Auscultatory); 2, Regression line (Dinamap).

( $r=0.23)$, height $(r=0.22)$ and weight $(r=0.2)$. However, combination of all the variables in a stepwise multiple regression analysis showed that a model only including CBP (model $r^{2}=0.49$ ) could not be further improved by the inclusion of age or the indices of body size, suggesting that the correlation of WCE with body size is likely due to its covariation with CBP.

The residual difference to daytime ABPM was less strongly correlated with the prevailing HBP $(r=0.42, p<0.0001)$ than with CBP (see Fig. 2B). Thus in contrast to CBP, HBP was equally reliable throughout the whole range of measurements. However, age $(r=0.19, p<0.05)$ and body size (height $r=$ 0.27 , weight $r=0.25$; all $p<0.05$ ) also affected the deviation of HBP from daytime ABPM.

Table 2. Accuracy and precision of $A B P M$ prediction by $H B P$ and $C B P$

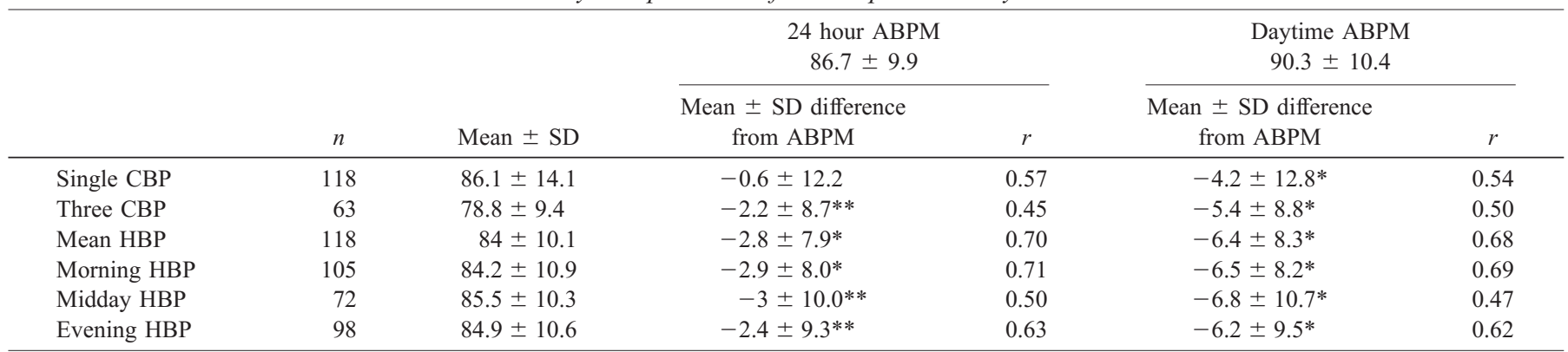

All blood pressures are given as mean arterial pressure (MAP) in mmHg. $r=$ Spearman correlation coefficient.

Significance of difference from zero: $* p<0.01,{ }^{*} p<0.05$. 
Sensitivity and specificity of HBP and CBP in diagnosing hypertension

Figure 3 illustrates the level of agreement of the three methods in identifying hypertensive patients. While $23 \%$ of observations were classified as hypertensive by daytime ABPM and $25 \%$ by HBP, $39 \%$ of the CBP measurements were above the 95th percentile. The rate of false positives was also lower for HBP at $13 \%$ compared with $23 \%$ with CBP. The resulting sensitivity and specificity rates of detecting hypertension with HBP and CBP are given in Table 3. HBP had a higher specificity, but a lower sensitivity than CBP in detecting hypertensive subjects. The combination of CBP and HBP measurements could increase diagnostic sensitivity and specificity. Sensitivity increased to $81 \%$ when the diagnosis of hypertension was made based on an elevation of either CBP or HBP; however this resulted in a loss of specificity. When abnormal values both in the clinic and at home were used to classify a patient as hypertensive, specificity increased to $92 \%$ and the positive predictive value to $61 \%$, but only $41 \%$ of all truly hypertensive subjects were diagnosed correctly.

\section{Effects of timing and experience on HBP accuracy and precision}

In a group of 55 patients in whom HBP readings were obtained on each of the 3 days before and after ABPM, we examined over which time periods the best prediction of ABPM could be achieved. Averaging HBP over 6 days, compared with four ( 2 days immediately before and after ABPM) and 2 days ( 1 day immediately before and after ABPM), resulted mainly in an increase of sensitivity (2 d: $44 \%, 4 \mathrm{~d}$ : $55 \%, 6$ d: $67 \%)$, while specificity remained similar $(87 \%$ to $89 \%$ ). The correlation of HBP to daytime ABPM was $r=0.70$ for a 2-day period, $r=0.73$ for 4 days and $r=0.72$ for 6 days. Notably, the mean difference between ABPM and HBP did not change significantly when different numbers of days were compared, indicating a systematic difference between ABPM and HBP.

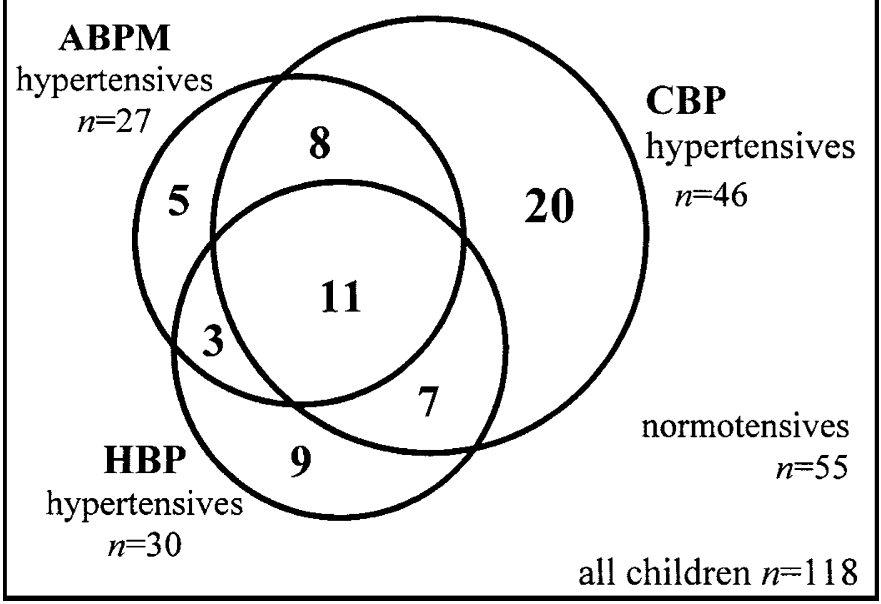

Figure 3. Overlap of hypertension as defined by daytime ABPM, CBP and HBP. Circles represent patients classified as hypertensive by different methods and their overlap.
Table 3. Sensitivity and specificity of $H B P$ and $C B P$ for detecting hypertension (daytime $A B P M$ as reference)

\begin{tabular}{lcccc}
\hline & & & \multicolumn{2}{c}{ Predictive value } \\
\cline { 4 - 5 } & Sensitivity & Specificity & Positive & Negative \\
\hline CBP & 0.7 & 0.7 & 0.41 & 0.88 \\
HBP & 0.52 & 0.82 & 0.47 & 0.85 \\
CBP or HBP & 0.81 & 0.60 & 0.38 & 0.92 \\
CBP and HBP & 0.41 & 0.92 & 0.61 & 0.84 \\
Mean of CBP \& HBP & 0.63 & 0.87 & 0.59 & 0.89 \\
\hline
\end{tabular}

To examine whether the precision of HBP changed with longer term use we analyzed data for two consecutive visits, which were at least six months apart, in a subgroup of 70 patients. The MAP as measured by 24 hour ABPM decreased from the first visit $(87.4 \pm 9.1 \mathrm{~mm} \mathrm{Hg})$ to the second visit $(83.2$ $\pm 7.4 \mathrm{~mm} \mathrm{Hg}$ ), due to an increased percentage of children on antihypertensive medication (first visit: $81 \%$, second visit: $96 \%$ ). While ABPM diagnosed $24 \%$ and $11 \%$ of children as hypertensive on the first and second visit respectively, the fraction of patients rated hypertensive by HBP decreased from $27 \%$ to $9 \%$. The specificity of detecting hypertension increased (first visit: $79 \%$, second visit: $94 \%$ ), whereas sensitivity dropped from $47 \%$ to $21 \%$. The $95 \%$ range of agreement with ABPM improved slightly from 36 to $32 \mathrm{~mm} \mathrm{Hg}$, while the mean difference between HBP and ABPM did not change significantly. The correlation between HBP and 24 hour ABPM remained constant over time (first visit: $r=0.61$, second visit $r=0.6$ ).

\section{White coat hypertension}

Twenty-seven children in this study had white coat hypertension $(\mathrm{WCH})$, i.e. $\mathrm{CBP}$ above the $95^{\text {th }}$ percentile but daytime ABPM below the $95^{\text {th }}$ percentile. Both systolic and diastolic CBP were high in $17 \%$ of these children while selective systolic $\mathrm{WCH}$ was observed in $9 \%$ and selective diastolic $\mathrm{WCH}$ in $74 \%$. Of the 46 children presenting hypertensive in clinic 59\% had a normal ABPM. However CBP also failed to identify 8 of the 27 children who did have ABPM hypertension, i.e. "white coat normotension" was observed in $5 \%$ of all children and in $29 \%$ of all truly hypertensive children. There were no significant differences between children with and without WCH in respect to casual or ambulatory heart rate, sex distribution, age, body dimensions or type, severity, duration and treatment of renal disease. Also, we were unable to identify independent predictors for the occurrence of white coat hypertension in a logistic regression model.

In a more comprehensive assessment of CBP we included two additional readings around the original visit if antihypertensive medication had remained unchanged over this period. When the three consecutive CBP readings were averaged, only $6.3 \%$ of children still had WCH. With the use of three CBP measurements the specificity of CBP in detecting hypertension increased from $70 \%$ to $93 \%$, but sensitivity fell slightly from $70 \%$ to $67 \%$. Similarly to HBP, the mean absolute difference from daytime ABPM was not changed significantly by the use of three repeated measurements. 
Notably, the addition of HBP to three CBP readings did not result in any increase in sensitivity, i.e. identified no additional truly hypertensive children (see Table 4). A specificity of $98 \%$ could be reached if only children in whom mean HBP and all three CBP readings were abnormal were considered hypertensive, but in this case sensitivity was rather poor at $33 \%$.

\section{DISCUSSION}

The increasing availability of self-blood pressure monitoring equipment has lead to increasing interest in the subject and the development of detailed application guidelines for adults (12, 13). However, data on HBP in children are very limited, and it is unclear how much specific pediatric issues, such as the effect of age, cuff size and difficulty in obtaining cooperation of children influence the validity of this technique. Our study is the first to systematically examine the concordance of ABPM, CBP and HBP in a large pediatric cohort (13a).

In adults ABPM has become generally accepted as gold standard due to its superior prediction of end organ damage (3), better reproducibility (14), ability to detect white coat hypertension and important relation to cardiovascular morbidity in patients with chronic renal failure (4). Throughout our study we also used ABPM as the method of reference even though data on end organ damage in children are not yet available. However ABPM in children has also been shown to have superior reproducibility to CBP (15) and is the preferred way of determining the size of the white coat effect.

In our study population of children with mild to moderate renal failure HBP achieved a specificity of $92 \%$ and a sensitivity of $50 \%$ for the detection of hypertension when using ABPM as gold standard. These values are in a range comparable to those recently reported for home self-measurements by adult patients (16-18). Relative to a single CBP measurement HBP had greater specificity while CBP had greater sensitivity. HBP and CBP could be used in different combinations to increase either sensitivity or specificity, but not both. A maximum specificity of $96 \%$ was reached when only a high HBP and CBP were sufficient to diagnose hypertension, but sensitivity remained low at $50 \%$. Using HBP in addition to three combined clinic readings did also not result in any increase in sensitivity, but could only increase specificity. We therefore suggest that, due to the better specificity than sensitivity of HBP, its best use may be as a diagnostic test for the exclusion of hypertension after a high CBP reading. In this setting a test with high specificity helps to avoid the unnecessary investigation and treatment of what is often white coat hypertension.

Table 4. Sensitivity and specificity of $H B P$ and three $C B P(2$ months before and 2 months after daytime ABPM and HBP measurements) for detecting hypertension

\begin{tabular}{lcccc}
\hline & & & \multicolumn{2}{c}{ Predictive value } \\
\cline { 3 - 5 } & Sensitivity & Specificity & Positive & Negative \\
\hline 3 CBP & 0.67 & 0.93 & 0.33 & 0.98 \\
3 CBP or HBP & 0.67 & 0.90 & 0.25 & 0.98 \\
3 CBP and HBP & 0.33 & 0.98 & 0.5 & 0.97 \\
Mean of 3 CBP \& HBP & 0.67 & 0.93 & 0.33 & 0.98 \\
\hline$n=63$. & & & &
\end{tabular}

CBP on the other hand, with its greater sensitivity, may be more suited for use as a screening test (19). This interpretation is in line with the recommendations of the Consensus Conference on self-blood pressure measurement for adults (13). In patients with established hypertension however, HBP has additional benefits through providing a greater number of readings that can help to evaluate changes in the efficacy of antihypertensive treatment in between clinical visits. In addition data from adult patients suggest that regular selfmeasurement of the blood pressure improves responder rates to antihypertensive medication (20), however false recording by the patient can introduce another source of error (21).

A problem for the exact determination of the sensitivity and specificity of HBP is the lack of established reference values for children. For our analysis we used normative data established for CBP (9) since HBP is also taken at rest. However the systematic difference of mean HBP from CBP in our study, which appears to be due to the white coat phenomenon, suggests that the use of CBP normative data may be inappropriate to determine hypertension by use of HBP. As HBP readings are generally lower, the use of $\mathrm{CBP}$ reference values may in part be responsible for the apparently lower sensitivity of HBP. Alternatively daytime ABPM reference values could have been used, but this was felt inappropriate since ABPM measurements are affected by increased physical activity during the day (22). To our knowledge there has been no attempt so far to define normative HBP data on healthy children. A similar debate is currently ongoing for the adult population (23).

Correlation coefficients may provide a surrogate measure for the quality of HBP measurements, because they are independent of absolute values. Correlation between HBP and ABPM in our study was slightly lower, but correlation of ABPM and CBP was similar, to those reported in well-controlled adult studies $(18,24)$. The superior strength of correlation of HBP to daytime and 24 hour ABPM compared with CBP in our study suggests that HBP may have a better accuracy in predicting ABPM in children. This was also supported by the markedly narrower 95\% range of agreement with ABPM (HBP: $33 \mathrm{~mm}$ $\mathrm{Hg}$; CBP: $51 \mathrm{~mm} \mathrm{Hg}$ ).

Casual blood pressure measurements are known to be susceptible to the white coat effect (WCE), which makes the interpretation of high values less certain. We noted a close correlation between absolute CBP values and the difference between CBP and ABPM, confirming previous findings in adults (17) and children (25). This effect was not significantly dependent on the method used to measure CBP and was much less pronounced for HBP. Therefore, in contrast to CBP, HBP readings are not less accurate in the high range of blood pressure. This suggests that HBP readings may be largely independent of an alerting response related to the measurement itself, which would resemble the white coat effect.

To date there are no recommendations regarding when and how frequently HBP should be measured in children. Our data allow the suggestion that morning readings may be more reliable than evening measurements. By any means, the largest number of readings, taken both over several days and at different times of day, yielded the best prediction of ABPM. 
Extended use did not appear to significantly improve the accuracy of HBP, though the $95 \%$ limit of agreement with ABPM decreased slightly in width from 36 to $32 \mathrm{~mm} \mathrm{Hg}$. However, over the time period of our analysis there might have been too much change in antihypertensive medication to provide conclusive evidence.

In conclusion, HBP appears to be a valuable addition to $\mathrm{CBP}$, as it agrees with ABPM more closely and more consistently over the whole range of BP. When HBP is used in combination with CBP a higher degree of diagnostic specificity can be achieved than with CBP alone. The accuracy and precision of HBP remains consistent when applied over an extended time period. However our data do not support the replacement of ABPM by HBP. Firstly, the maximum diagnostic sensitivity reached by HBP and CBP was only $81 \%$, thus one out of four children diagnosed as hypertensive by ABPM would have been missed, even when both CBP and HBP were used in combination. Secondly, the range of agreement of HBP with ABPM, albeit narrower than that of CBP, is unacceptably wide. Thirdly, disruption of the diurnal BP regulation at night, which has a high prevalence in children with CRF $(13 a, 26)$ and is an independent risk factor for the development of left ventricular hypertrophy in CRF (27), cannot be assessed by HBP.

\section{APPENDIX}

\section{Members of the ESCAPE (Effect of Strict Blood Pressure} Control and ACE Inhibition on the Progression of CRF in Pediatric Patients) Trial Group. A. Anarat (Adana), A. Bakkaloglu (Ankara), A. Peco-Antic (Belgrade), U. Querfeld, J. Gellermann (Berlin), P. Sallay (Budapest), D. Drozdz (Crakow), K.-E. Bonzel, A.-M. Wingen (Essen), A. Zurowska (Gdansk), F. Perfumo, A. Canepa (Genoa), D.E. MüllerWiefel, K. Zepf (Hamburg), G. Offner, B. Enke (Hannover), O. Mehls, F. Schaefer, E. Wühl, C. Hadtstein (Heidelberg), U. Berg, G. Celsi (Huddinge), S. Emre, A. Sirin, I. Bilge (Istanbul), S. Çaliskan (Istanbul-Cerrahpasa), S. Mir, E. Serdaroglu (Izmir), C. Greiner, H. Eichstädt (Leipzig), K. HohbachHohenfellner (Mainz), N. Jeck (Marburg), A. Appiani, G. Ardissino, S. Testa (Milano), G. Montini (Padova), P. Niaudet, M. Charbit (Paris), J. Dusek (Prague), A. Caldas-Afonso (Porto), S. Picca, C. Matteucci (Rome), M. Wigger (Rostock), M. Fischbach, J. Terzic (Strasbourg), J. Fydryk, T. Urasinski (Szezecin), R. Coppo, L. Peruzzi (Torino), A. Jankauskiene (Vilnius), M. Litwin, M. Abuauba, R. Grenda (Warszawa), K. Arbeiter (Vienna), T.J. Neuhaus (Zurich).

\section{REFERENCES}

1. Klahr S, Levy AD, Beck GJ 1994 The effects of dietary protein restriction and blood-pressure control on the progression of chronic renal disease. N Engl J Med 330:877-884

2. Wingen AM, Fabian-Bach C, Schaefer F, Mehls O 1997 Randomised multicentre study of a low-protein diet on the progression of chronic renal failure in children.
European Study Group of Nutritional Treatment of Chronic Renal Failure in Childhood Lancet 349:1117-1123

3. Mancia G, Parati G 2000 Ambulatory blood pressure monitoring and organ damage. Hypertension 36:894-900

4. Liu M, Takahashi H, Morita Y, Maruyama S, Mizuno M, Yuzawa Y, Watanabe M, Toriyama T, Kawahara H, Matsuo S 2003 Non-dipping is a potent predictor of cardiovascular mortality and is associated with autonomic dysfunction in haemodialysis patients Nephrol Dial Transplant 18:563-569

5. Mule G, Caimi G, Cottone S, Nardi E, Andronico G, Piazza G, Volpe E, Federico MR, Cerasola G 2002 Value of home blood pressures as predictor of target organ damage in mild arterial hypertension. J Cardiovasc Risk 9:123-129

6. Chobanian AV, Barkis GL, Black DL, Cushman WC, Green LA, Izzo JLJ, Jones DW, Materson BJ, Oparil S, Wright JTJ, Roccella EJ 2003 The Seventh Report of the Joint National Committee on Prevention, Detection, Evaluation, and Treatment of High Blood Pressure: The JNC 7 Report. JAMA 289:2560-2571

7. Barker ME, Shiell AW, Law CM 2000 Evaluation of the Dinamap 8100 and Omron M1 blood pressure monitors for use in children. Paediatr Perinat Epidemiol 14:179186

8. Wühl E, Witte K, Soergel M, Mehls O, Schaefer F, Kirschstein M, Busch C, Danne T, Gellermann J, Holl R, Krull F, Reichert H, Rascher W, Reusz GS, German Working Group on Pediatric Hypertension 2002 Distribution of 24-h ambulatory blood pressure in children: normalized reference values and role of body dimensions J Hypertens 20:1995-2007

9. de Man SA, André JL, Bachmann HJ, Grobbee DE, Ibsen KK, Laaser U, Lippert P, Hofmann A 1991 Blood pressure in childhood: pooled findings of six European studies. J Hypertens 9:109-114

10. Zuther P, Witte K, Lemmer B 1996 ABPM-Fit and CV-Sort: an easy-to-use software package for detailed analysis of data from ambulatory blood pressure monitoring. Blood Press Monit 1:347-354

11. Bland JM, Altman DG 1986 Statistical methods for assessing agreement between two methods of clinical measurement. Lancet I:307-310

12. White WB, Asmar R, Imai Y, Mansoor G, Padfield P, Thijs L, Waeber B 1999 Task Force VI: Self-monitoring of the blood pressure. Blood Press Monit 4:343-351

13. Herpin D, Pickering TG, Stergiou GS, de Leeuw PW, Germano G 2000 Consensus Conference on Self-blood pressure measurement. Clinical applications and diagnosis. Blood Press Monit 5:131-135

13a. Lingens N, Freund M, Seeman T, Witte K, Lemmer B, Scharerk K 1997 Circadian blood pressure changes in untreated children with kidney disease and conserved renal function. Acta Pediatr 86:719-723

14. James GD, Pickering TG, Yee LS, Harshfield GA, Riva S, Laragh JH 1988 The reproducibility of average ambulatory, home and clinic pressures. Hypertension 11:545-549

15. Lurbe E, Redon J 2002 Reproducibility and validity of ambulatory blood pressure monitoring in children. Am J Hypertens 15:69S-73S

16. Stergiou GS, Zourbaki AS, Skeva II, Mountokalakis TD 1997 White coat effect detected using self-monitoring of blood pressure at home: comparison with ambulatory blood pressure. Am J Hypertension 11:820-827

17. Hond ED, Celis H, Fagard R, Keary L, Leeman M, O'Brien E, Vandenhoven G, Staessen JA, THOP investigators 2003 Self-measured versus ambulatory blood pressure in the diagnosis of hypertension. J Hypertens 21:717-722

18. Masding MG, Jones JR, Bartley E, Sandeman DD 2001 Assessment of blood pressure in patients with Type 2 diabetes: comparison between home blood pressure monitoring, clinic blood pressure measurement and 24-h ambulatory blood pressure monitoring. Diabet Med 18:431-437

19. Stergiou GS, Efstathiou SP, Argyraki CK, Gantzarou AP, Roussias LG, Mountokalakis TD 2002 Clinic, home and ambulatory pulse pressure: comparison and reproducibility. J Hypertens 20:1987-1993

20. Vetter W, Hess L, Brignoli R 2000 Influence of self-measurement of blood pressure on the responder rate in hypertensive patients treated with losartan: results of the SVATCH study. Standard vs Automatic Treatment Control of COSAAR in hypertension. J Hum Hypertens 14:235-241

21. Nordman A, Frach B, Walker T, Martina B, Battegay E 2000 Comparison of self-reported home blood pressure measurements with automatically stored values and ambulatory blood pressure. Blood Press 9:200-205

22. Bald M, Hoyer PF 2001 Measurement of blood pressure at home: survey among pediatric nephrologists. Pediatr Nephrol 16:1058-1062

23. Verdeccia P 2001 Reference values for ambulatory blood pressure and self-measured blood pressure on prospective outcome data. Blood Press Monit 6:323-327

24. Jula A, Puukka P, Karanko H 1999 Multiple clinic and home blood pressure measurements versus ambulatory blood pressure monitoring. Hypertension 34:261266

25. Matsuoka S, Kawamura K, Honda M, Awazu M 2002 White coat effect and white coat hypertension in pediatric patients. Pediatr Nephrol 17:950-953

26. Mitsnefes MM, Kimball TR, Daniels SR 2003 Office and ambulatory blood pressure elevation in children with chronic renal failure. Pediatr Nephrol 18:145-149

27. Covic A, Goldsmith DJ, Covic M 2000 Reduced blood pressure diurnal variability as a risk factor for progressive left ventricular dilatation in hemodialysis patients. Am J Kidney Dis 35:617-623 